\title{
Antioxidants to Prevent Bovine Neutrophil-Induced Mammary Epithelial Cell Damage*
}

\author{
K. Lauzon, ${ }^{1,2}$ X. Zhao, ${ }^{2}$ A. Bouetard, ${ }^{1}$ L. Delbecchi, ${ }^{1}$ B. Paquette,${ }^{3}$ and P. Lacasse ${ }^{1}$ \\ ${ }^{1}$ Dairy and Swine Research and Development Centre, Agriculture and Agri-Food Canada, \\ Lennoxville, Québec, Canada J1M 1 Z3 \\ 2Department of Animal Science, McGill University, Ste-Anne de Bellevue, Québec, Canada H9X 3V9 \\ ${ }^{3}$ Department of Nuclear Medicine and Radiobiology, Faculty of Medicine, Université de Sherbrooke, \\ Québec, Canada, J1H 5N4
}

\begin{abstract}
Activated neutrophils are able to produce a large quantity of bactericidal molecules such as reactive oxygen species that have been associated with tissue damage in several inflammation models. The protective effects of antioxidants in a context of neutrophil-induced damage to mammary epithelial cells were first evaluated in vitro using a coculture model of activated bovine neutrophils and a bovine mammary epithelial cell line (MAC-T cells). Cell damage was determined by quantifying the release of lactate dehydrogenase by MAC-T cells in culture medium. Morphological observation of cells stained with acridine orange was used to visualize the extent of cell damage. When incubated with neutrophils activated by lipopolysaccharides and phorbol 12myristate 13-acetate, MAC-T cells released large amounts of lactate dehydrogenase indicating significant cell damage. The addition of dimethylthiourea or bathocuproine disulfonic acid did not reduce the damage whereas catechin, deferoxamine or glutathione ethyl ester significantly reduced neutrophil-induced cytotoxicity in a dose-dependent manner. The effect of deferoxamine, an iron chelator, on the growth of Escherichia coli and the ability of bovine neutrophils to phagocytose these bacteria were then assessed in vitro. Our data showed that deferoxamine did not interfere with the phagocytic activity of neutrophils but inhibited growth of the bacteria. Overall, our results suggest that antioxidants may be effective tools for protecting mammary tissue against neutrophil-induced oxidative stress during bovine mastitis.
\end{abstract}

(Key words: mastitis, inflammation, antioxidant, reactive oxygen species)

Abbreviation key: BDA = bathocuproine disulfonic acid, CAT = catechin, DFO = deferoxamine, DMEM =

Received May 3, 2005.

Accepted July 29, 2005.

Corresponding author: Pierre Lacasse; e-mail: lacassep@agr.gc.ca.

*Dairy and Swine Research and Development Centre contribution no. 867 .
Dulbecco's modified Eagle medium, DMTU = dimethylthiourea, FBS = fetal bovine serum, GEE = glutathione ethyl ester, GSH = glutathione, $\mathbf{L D H}=$ lactate dehydrogenase, PMA = phorbol 12-myristate 13-acetate, PMN = polymorphonuclear neutrophils, ROS = reactive oxygen species.

\section{INTRODUCTION}

The economic losses caused by mastitis are well known in the dairy industry. Mastitis is an inflammatory reaction that usually occurs following an IMI. Local recruitment of immune cells such as macrophages and neutrophils (PMN) is a crucial step in the development of an acute inflammatory response. Neutrophils are essential for effective host defense but they are also known to be involved in various inflammatory diseases (Weiss, 1989; Smith, 1994). Activated PMN reach the infected site and release toxic reactive oxygen species (ROS) via an oxygen-dependent pathway. The cornerstone of this process is the generation of superoxide anion $\left(\mathrm{O}_{2}^{-}\right)$via the enzyme NADPH-oxidase, which leads to the production of other ROS such as hydrogen peroxide $\left(\mathrm{H}_{2} \mathrm{O}_{2}\right)$, hydroxyl radical $\left(\mathrm{OH}^{\circ}\right)$, and hypochlorous acid (HOCl). Although these ROS are harmful to bacteria, they also have the potential to destroy host cells. Several studies on the inflammation process have shown that oxidants released by PMN induce tissue damage (Weiss, 1989; van Asbeck, 1990). Oxidants are known to be cytotoxic through many different mechanisms such as protein and amino acid oxidation, lipid peroxidation, and DNA damage. Some ROS can play a role in the transmission of intracellular signals.

Recently, it was demonstrated in vitro that activated blood PMN have a cytotoxic effect on mammary epithelial cells (Ledbetter et al., 2001) potentially through the release of ROS such as hydroxyl radicals (Boulanger et al., 2002). In cases of acute mastitis such as those caused by Escherichia coli, the amount of ROS released by PMN may overwhelm the cow's endogenous antioxidant protection mechanisms and therefore add to the inflammation causing extensive tissue damage. There- 
fore, it is plausible that supplementation with exogenous antioxidants may facilitate the cow's recovery from mastitis and protect the secretory epithelial cells.

The small pool of cellular iron that is loosely bound to cellular constituents is believed to participate in the generation of highly reactive oxygen species (Dehne et al., 2001). When deferoxamine (DFO) chelates free iron, it forms ferrioxamine, which is a very stable complex distributed in the extracellular space and unable to penetrate cells (Emerit et al., 2001). Gannon et al. (1987) demonstrated that pretreating cultured bovine pulmonary artery endothelial cells with DFO decreases PMN-mediated injury. Thus, the use of this chelator may prevent the Fenton reaction and generation of hydroxyl radicals. Dimethylthiourea (DMTU) is a hydroxyl radical and hydrogen peroxide scavenger that is able to inactivate ROS (Sprong et al., 1997; Visseren et al., 2002). Protective effects of DMTU were found in several models of acute lung injury induced by phorbol 12-myristate 13-acetate (PMA). Cuprous ions are also thought to be strong pro-oxidants that form radicals by a Fenton-type reaction or by transition complexes with molecular oxygen. Therefore, elimination of these ions by chelators such as bathocuproine disulfonic acid (BDA) could lower the oxidizing effects of various ROS. Oikawa and Kawanishi (1996) showed that BDA completely prevented DNA damage that was likely mediated by $\mathrm{H}_{2} \mathrm{O}_{2}$. Furthermore, using a human breast carcinoma cell line, Verhaegh et al. (1997) demonstrated in vitro that BDA prevented hydroxyl radical formation. Catechin (CAT) is a flavonoid polyphenol extracted from plants. It has oxygen-scavenging properties and anti-inflammatory effects (Yang et al., 1998). Glutathione (GSH) is a key component of the cellular defense cascade against injury caused by ROS (Kennedy and Lane, 1994). It is the key substrate of the endogenous $\mathrm{H}_{2} \mathrm{O}_{2}$-removing enzyme GSH peroxidase. It is also a scavenger of hydroxyl radicals and singlet oxygen. Reduced glutathione ethyl ester (GEE) is a cellular antioxidant that can easily cross membranes and help to maintain the intracellular GSH concentration.

In this study, a coculture model composed of MACT cells (Huynh et al., 1991) and bovine PMN was used to evaluate the effects of selected antioxidants and chelators on PMN-induced damage of mammary epithelial cells. The effect of DFO on bacterial growth and on the ability of PMN to ingest $E$. coli was also investigated.

\section{MATERIALS AND METHODS}

\section{Chemicals and Reagents}

Unless otherwise specified, all the reagents used were purchased from Sigma Chemical Co. (St. Louis, MO). Lipopolysaccharide (Escherichia coli O55:B5), DFO,
GEE, DMTU, and BDA were dissolved in Hanks' balanced salt solution. Catechin was dissolved in $100 \%$ ethanol before being further diluted in Hanks' balanced salt solution. Phorbol 12-myristate 13-acetate was dissolved in dimethyl sulfoxide.

\section{Cell Culture and Coculture}

The bovine mammary epithelial cell line MAC-T was maintained in culture by serial passages in $75-\mathrm{cm}^{2}$ flasks at $37^{\circ} \mathrm{C}$ in a humidified atmosphere containing $5 \% \mathrm{CO}_{2}$. The routine culture medium was composed of complete Dulbecco's modified Eagle's medium (DMEM) supplemented with 10\% fetal bovine serum (FBS; HyClone, Logan, UT) and 1\% (vol/vol) antibiotic/antimycotic (Sigma).

Neutrophils were isolated from healthy midlactating Holstein cows using a method described by Carlson and Kaneko (1973) with some modifications. Blood samples were collected from the caudal vein into EDTA-coated Vacutainer tubes (Becton Dickinson Co., Franklin lakes, NJ). Whole blood was diluted 1:1 with prewarmed $\left(37^{\circ} \mathrm{C}\right)$ Hanks' balanced salt solution and carefully layered over $20 \mathrm{~mL}$ of Ficoll-Paque Plus (Amersham Pharmacia, Montreal, QC, Canada) in 50-mL Falcon tubes. The tubes were then centrifuged at $1000 \times$ $g$ for 40 min at $4^{\circ} \mathrm{C}$, after which the plasma and Ficoll were gently removed and discarded. The PMN were then isolated by 2 hypotonic lyses of the erythrocyte pellet using $15 \mathrm{~mL}$ of Tris-buffered $0.15 \mathrm{M}$ ammonium chloride. The cells were washed twice in Hanks' balanced salt solution using centrifugation at $350 \times g$ for $10 \mathrm{~min}$ before being resuspended at a concentration of $5 \times 10^{6} \mathrm{PMN} / \mathrm{mL}$ in the cytotoxicity medium $[47.5 \%$ phenol red-free RPMI 1640 medium, $47.5 \%$ phenol redfree DMEM, 2.5\% very-low endotoxin FBS (HyClone), $1 \%$ antibiotic/antimycotic solution, $5 \mu \mathrm{g} / \mathrm{mL}$ of bovine insulin, $2 \mathrm{~m} M$ L-glutamine, and $1.4 \mathrm{mg}$ of D-(+)-glucose/ $\mathrm{mL}$. Cell viability was evaluated using trypan blue dye exclusion and normally exceeded $95 \%$ in this study. More than $95 \%$ of the cells isolated were neutrophils.

Coculturing of bovine PMN and MAC-T cells was performed as described by Boulanger et al. (2002). Briefly, epithelial cells were grown in the cytotoxicity medium on 24 -well plates $(\sim 50,000$ cells/well) precoated with a collagen matrix (see below). Once the epithelial cells were $80 \%$ confluent, the medium was removed and freshly isolated PMN were put into each well $\left(2.5 \times 10^{6}\right.$ PMN/well). Lipopolysaccharide (1 $\mu \mathrm{g} / \mathrm{mL})$ and PMA (1 $\mu \mathrm{g} / \mathrm{mL}$ ) were then added to each well in the presence or absence of various concentrations of tested antioxidants. All treatments were done in triplicate. Phorbol 12-myristate 13-acetate was used because it activates both pools of NADPH oxidase in PMN (plasma mem- 
brane and granule membranes) and induces extracellular release of oxygen metabolites (Karlsson et al., 2000). The plates were returned in the incubator for $24 \mathrm{~h}$. The substances evaluated in this study were DFO (0 to 300 $\mu M)$, CAT (0 to $75 \mu M)$, BDA (0 to $1000 \mu M)$, DMTU (0 to $10,000 \mu M$ ), and GEE (0 to $100 \mu M$ ).

\section{Cytotoxicity Assay}

After an incubation of $24 \mathrm{~h}$, the coculture supernatants were transferred into $1.5-\mathrm{mL}$ Eppendorf tubes and then centrifuged at $500 \times \mathrm{g}$ for $5 \mathrm{~min}$ in a microcentrifuge. The lactate dehydrogenase (LDH) assay was performed using the CytoTox96 Nonradioactive Cytotoxicity Assay kit (Promega, Madison, WI) according to the manufacturer's instructions and Fischer et al. (1998). The following controls were included in each assay: MAC-T cell spontaneous LDH release, PMN spontaneous LDH release, culture medium background, and MAC-T maximal release of LDH (15-min incubation of cells with lysis solution). For each experimental well, percentage cytotoxicity was calculated using the manufacturer's equations:

Eq. 1: Experimental well optical density - culture medium background optical density $=\mathrm{A}$

Eq. 2: MAC-T cell spontaneous LDH release optical density - culture medium background optical density $=\mathrm{B}$

Eq. 3: PMN spontaneous LDH release optical density - culture medium background optical density $=\mathrm{C}$

Eq. 4: MAC-T cell maximum LDH release optical density - volume correction optical density $=\mathrm{D}$

Eq. 5: $\%$ cytotoxicity $=(\mathrm{A}-\mathrm{B}-\mathrm{C}) /(\mathrm{D}-\mathrm{B}) \times 100$.

\section{Acridine Orange Staining of Cocultures}

Once supernatants were carefully collected for the LDH assay, the remaining cells were gently washed twice with Hanks' balanced salt solution before acridine orange staining solution ( $8 \mu \mathrm{g} / \mathrm{mL}$ in PBS) was added ( $200 \mu \mathrm{L} /$ well). The 24 -well plates were incubated for 10 min at room temperature. The acridine orange solution was then removed and the cells were fixed by adding $500 \mu \mathrm{L}$ of $4 \%$ (wt/vol) formaldehyde solution in PBS. Fluorescence was visualized using a fluorescence microscope (Hund Wilovert S, Wetzlar, Germany) and photographs were taken with a digital camera (Nikon, Coolpix 4500, Tokyo, Japan) coupled to the microscope.

\section{Preparation of Calf Collagen}

All procedures were conducted at 4 to $8^{\circ} \mathrm{C}$ as described by Miller and Rhodes (1982) with some modifications. Briefly, a skinless tail was immersed in $95 \%$ ethanol for 15 min to facilitate tendon detachment. All tendons were excised with a scalpel and placed in $95 \%$ ethanol. The tendons were dried under UV light overnight. The next morning, they were cut into 1-cm-long pieces and placed into $500 \mathrm{~mL}$ of $0.5 \mathrm{~N}$ acetic acid to extract collagen. The extraction was carried out for 3 to $4 \mathrm{~d}$ at $4^{\circ} \mathrm{C}$ with continuous vigorous shaking. The mixture was then filtered through several layers of cheesecloth to remove insoluble residues. Finally, the solution was centrifuged at $50,000 \times g$ for $45 \mathrm{~min}$ at $4^{\circ} \mathrm{C}$ to be clarified. The collagen solution was kept at $4^{\circ} \mathrm{C}$.

\section{Bacterial Growth Assay}

First, E. coli P4:O32 (H37, $\beta$-glucoronidase+, hemolysin-) were grown in $50 \mathrm{~mL}$ of brain-heart infusion medium for $2 \mathrm{~h}$ at $37^{\circ} \mathrm{C}$. An aliquot was then serially diluted to perform a bacteriological count (cfu) on tryptic soy agar plates that were incubated overnight at $37^{\circ} \mathrm{C}$. The $50-\mathrm{mL}$ culture was kept at $4^{\circ} \mathrm{C}$ overnight. The cells were then centrifuged at $50,000 \times g$ for 10 min, suspended in DMEM/F12 medium without phenol red at $2 \times 10^{7} \mathrm{cfu} / \mathrm{mL}$, and kept on ice until needed. In a 96-well plate containing DMEM /F12, DFO was added to obtain final concentrations of $0,50,200$, or $800 \mu M$ (in triplicate). Inactivated FBS was then added (5\% final concentration), and $125 \mu \mathrm{L}$ of the solution of bacteria was transferred into the wells (final volume of 250 $\mu \mathrm{L})$. Absorbance of the plate was then immediately read at $600 \mathrm{~nm}$ using a plate-reader; this was used as the time-zero reading. The plate was put in a shaking incubator at $37^{\circ} \mathrm{C}$ and absorbance was read every $15 \mathrm{~min}$ for $2 \mathrm{~h}$. The blank was composed of phenol red-free DMEM/F12 supplemented with 5\% FBS. The assay was repeated 3 times.

\section{Phagocytosis Assay}

Bacteria (E. coli P4) were prepared as described above at $4 \times 10^{7} \mathrm{cfu} / \mathrm{mL}$. One milliliter of bacteria was added to a tube containing $1 \mathrm{~mL}$ of freshly isolated $\operatorname{PMN}\left(2 \times 10^{6}\right)$ and DFO (final concentration: 0, 50, 200, or $800 \mu M$ ) in DMEM/F12 containing $5 \%$ inactivated FBS. The tubes were then transferred to a $37^{\circ} \mathrm{C}$ shaking bath and phagocytosis was allowed to proceed for 30 min. Another bacterial count was performed on an aliquot of the added solution of bacteria to determine the exact amount of bacteria added. At the end of the incubation, $100 \mu \mathrm{L}$ of suspension was taken from each tube and serially diluted in triplicate to perform a count 
(cfu). This count determines the number of bacteria remaining in the supernatant and hence the quantity of nonphagocytosed bacteria. By subtracting this number from the number of bacteria initially added, the number of ingested bacteria was determined. The assay was repeated 3 times.

\section{Statistical Analyses}

All data from the cytotoxicity assay and the phagocytosis assay were analyzed for statistical significance using the GLM procedure of SAS (version 9.1, 2002; SAS Institute, Inc., Cary, NC). The linear and quadratic effects of each antioxidant on cell cytotoxicity were assessed and the effects were considered significant at $P$ $\leq 0.05$ and tended to be significant at $0.05<P \leq 0.10$. When relevant, data are expressed as mean \pm SEM.

The effect of DFO on bacterial growth was subjected to repeated-measures ANOVA using the MIXED procedure of SAS. The covariance structure used was the spatial power and the model was:

$$
\begin{aligned}
& Y_{i j k}=\mu+D_{i}+T_{j}+A_{k}+\left(D_{i} \times A_{k}\right) \\
& +\left(D_{i} \times T_{j}\right)+\left(D_{i} \times T_{j} \times A_{k}\right)+\varepsilon_{i j k}
\end{aligned}
$$

where $Y_{i j k}$ is the variable studied (bacterial growth), $\mu$ is the overall mean, $\mathrm{D}_{\mathrm{i}}$ is the DFO-treatment effect, $\mathrm{T}_{\mathrm{j}}$ is the time effect, $\mathrm{A}_{\mathrm{k}}$ is the assay effect, and $\varepsilon_{\mathrm{ijk}}$ the residual error.

\section{RESULTS}

\section{Effect of PMN, Antioxidants, LPS, and PMA on Viability of MAC-T Cells}

Incubation of MAC-T cells with nonactivated bovine PMN $\left(5 \times 10^{6} / \mathrm{mL}\right)$ or LPS and PMA was not damaging to MAC-T cells after $24 \mathrm{~h}(P>0.1)$. When MAC-T cells were incubated with $25 \mu M$ CAT, $200 \mu M$ DFO, or 50 $\mu M$ GEE in the presence of LPS and PMA but without $\mathrm{PMN}$, the cytotoxicity levels were not significantly different from zero $(P>0.1)$, indicating that these antioxidants were not impairing cellular integrity. On the other hand, exposure of MAC-T cells to PMN activated by LPS and PMA resulted in a significant decrease of cell viability as revealed by the high levels of $\mathrm{LDH}$ released in supernatants (average cytotoxicity $=25.7 \pm$ $2.9 \%$ ). These results are consistent with those obtained by the acridine orange staining; MAC-T cells incubated with nonactivated PMN (Figure 1A) did not show any sign of cell death, and monolayer integrity was conserved, whereas nuclear condensation, abnormal cellular shapes, and disrupted monolayers were observed when the MAC-T cells were incubated with activated PMN (Figure 1B).

\section{Effect of Antioxidants on Neutrophil-Induced Mammary Cell Damage}

The effects of various antioxidants and chelators were evaluated using the coculture model of bovine PMN and MAC-T cells described above. When added to the cocultures of MAC-T cells and activated PMN, DMTU did not show any protective effect on PMN-induced cytotoxicity (data not shown). Addition of BDA reduced cytotoxicity levels by $33 \%(P<0.001)$ at the highest concentration (1000 $\mu M$; Figure 2A). On the other hand, CAT, DFO, and GEE decreased PMN-induced cytotoxicity in a dose-dependent manner. The addition of 25 and 50 $\mu M$ of CAT reduced cell cytotoxicity by 77 and $100 \%$, respectively $(P<0.001$; Figure $2 \mathrm{~B})$. Similarly, the addition of DFO at 100,200 , and $300 \mu M$ lowered cytotoxicity by 58,88 , and $98 \%$, respectively $(P<0.001$; Figure 2C). Addition of GEE at 50 and $100 \mu M$ decreased cytotoxicity levels by 82 and $83 \%$, respectively $(P<0.001$; Figure 2D). Protective effects of CAT, GEE, and DFO were confirmed by visual observation of cells stained with acridine orange under fluorescence microscopy (Figure 1, panels B through E).

\section{Bacterial Growth Assay}

The siderophore DFO can be used as a source of iron and promote growth of some bacteria (Diarra et al., 2002). Therefore, the effect of DFO on $E$. coli growth was evaluated using an in vitro assay. A significant reduction of bacterial growth $(P<0.05)$ was observed after a 60 -min incubation with DFO (Figure 3). Indeed, for all concentrations tested, the optical density of wells containing DFO was significantly lower than that of control for time points $60,75,90,105$, and $120 \mathrm{~min}(P$ $<0.05$ ).

\section{Phagocytosis Assay}

The release of free radicals is important for the functionality of PMN. Therefore, the effect of DFO on the ability of PMN to ingest $E$. coli was evaluated in vitro. A known amount of bacteria was added to different wells containing PMN $\left(2 \times 10^{6} \mathrm{PMN}\right)$ with or without DFO. Incubating $E$. coli in the DMEM/F12 medium for $30 \mathrm{~min}$ did not induce cell death (data not shown). Seventy-eight percent of the bacteria were ingested by PMN in control wells containing medium only. Deferoxamine did not affect $(P>0.4)$ the phagocytic ability of PMN, as shown in Figure 4. 

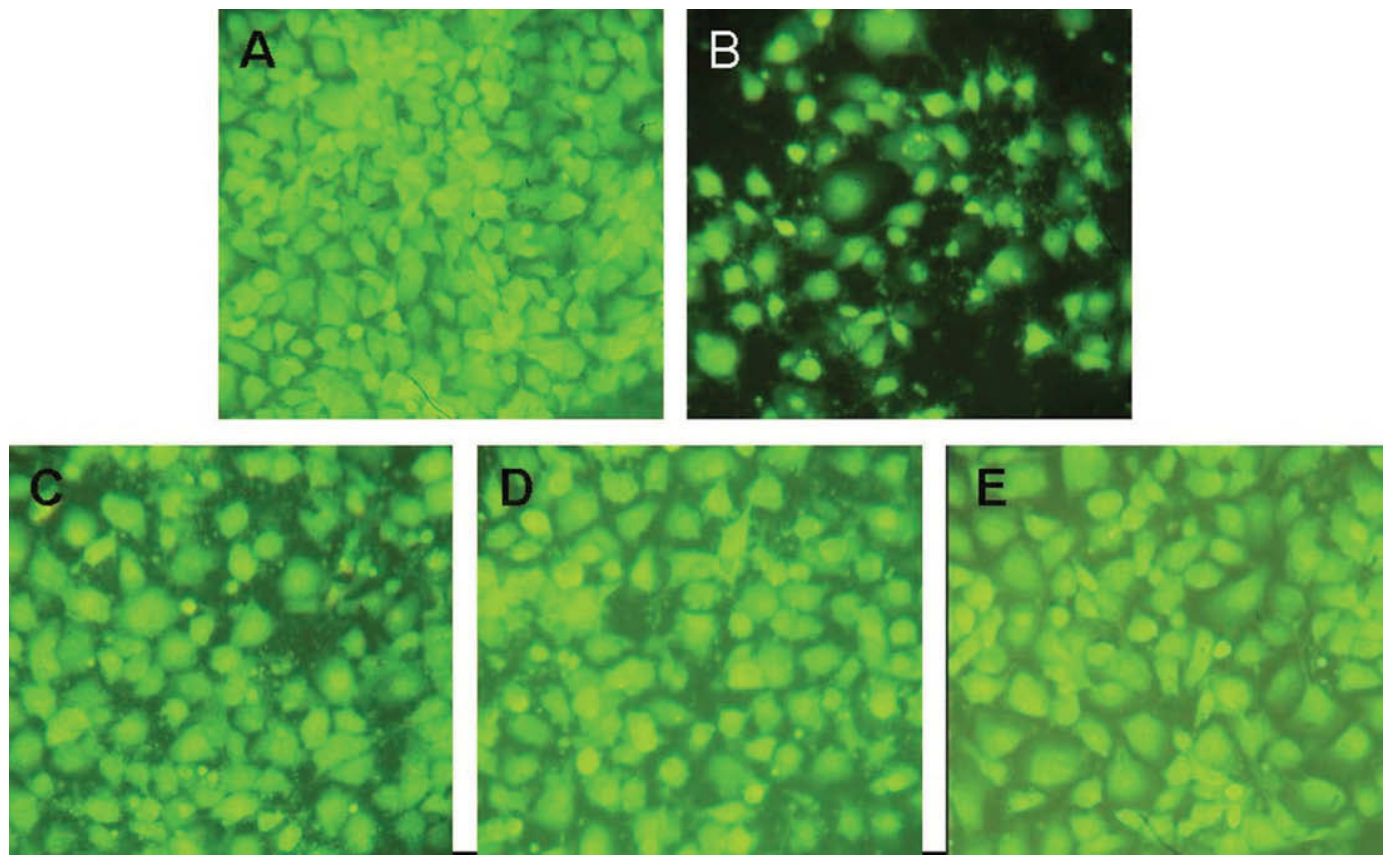

Figure 1. Typical acridine orange staining of MAC-T cells following incubation for $24 \mathrm{~h}$ with (A) nonactivated neutrophils $(\mathrm{PMN} ; 5 \times$ $10 \% / \mathrm{mL})$, (B) PMN activated with LPS $(1 \mu \mathrm{g} / \mathrm{mL})$ and phorbol 12-myristate 13 -acetate $(1 \mu \mathrm{g} / \mathrm{mL})$, (C) activated PMN and catechin $(25 \mu M)$, (D) activated PMN and deferoxamine $(200 \mu M)$, and (E) activated PMN with glutathione ethyl ester $(50 \mu M)$.
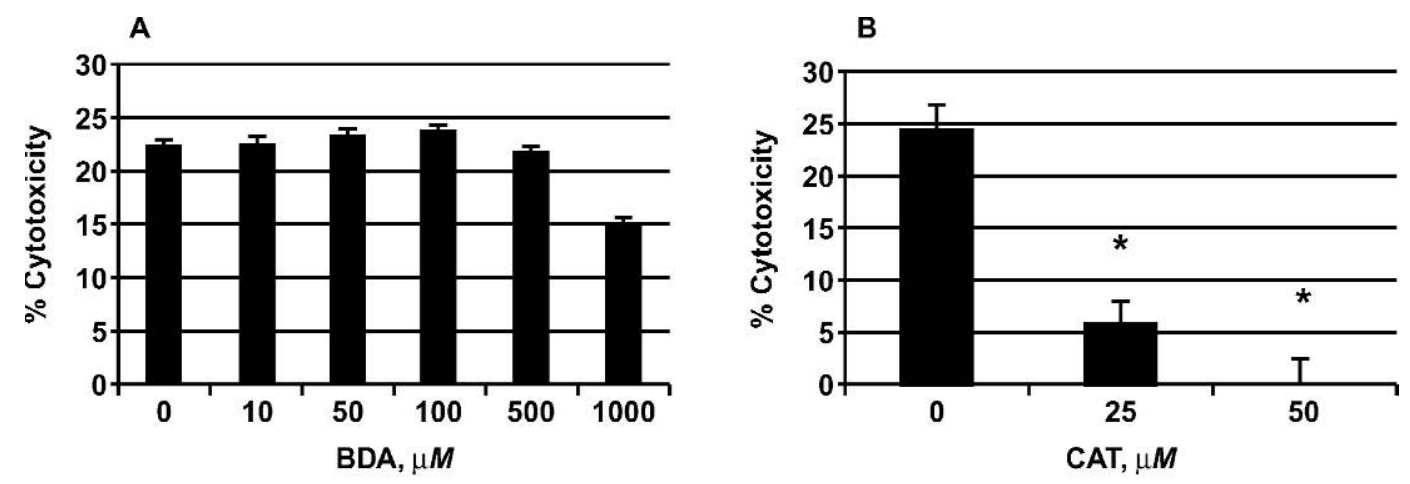

C
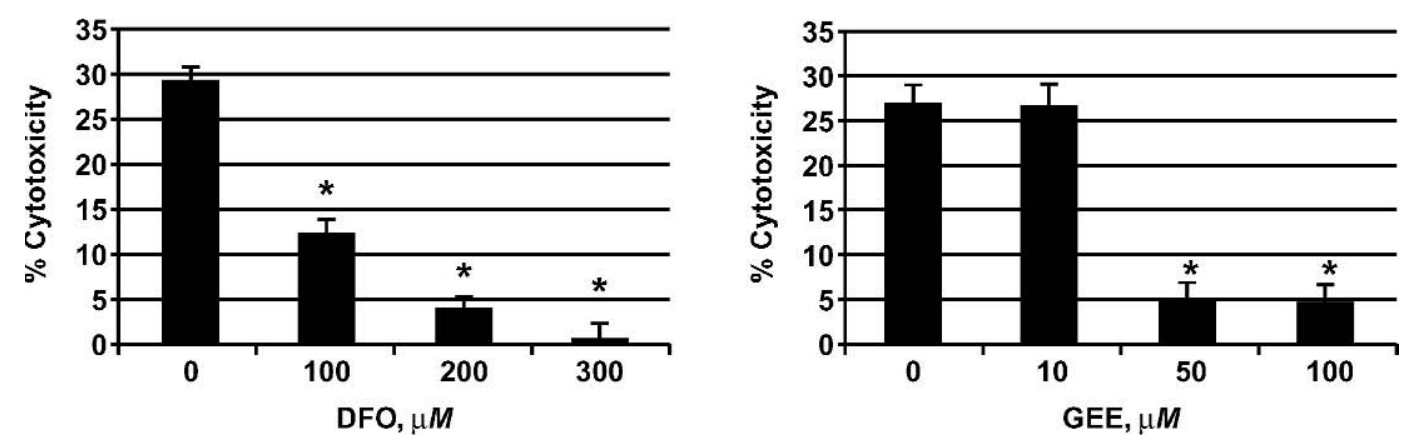

Figure 2. Levels of cellular damage in MAC-T cells coincubated with activated neutrophils (PMN; $\left.5 \times 10^{6} / \mathrm{mL}\right)$ in the presence of $(\mathrm{A})$ bathocuproine disulfonic acid (BDA), (B) catechin (CAT), (C) deferoxamine (DFO), and (D) glutathione ethyl ester (GEE). After $24 \mathrm{~h}$ of coincubation, cytotoxicity was determined by measuring of released lactate dehydrogenase. Data are expressed as percentage of cytotoxicity and represent LS means \pm SEM. *Value is significantly different $(P<0.05)$ from the control (no antioxidant). 


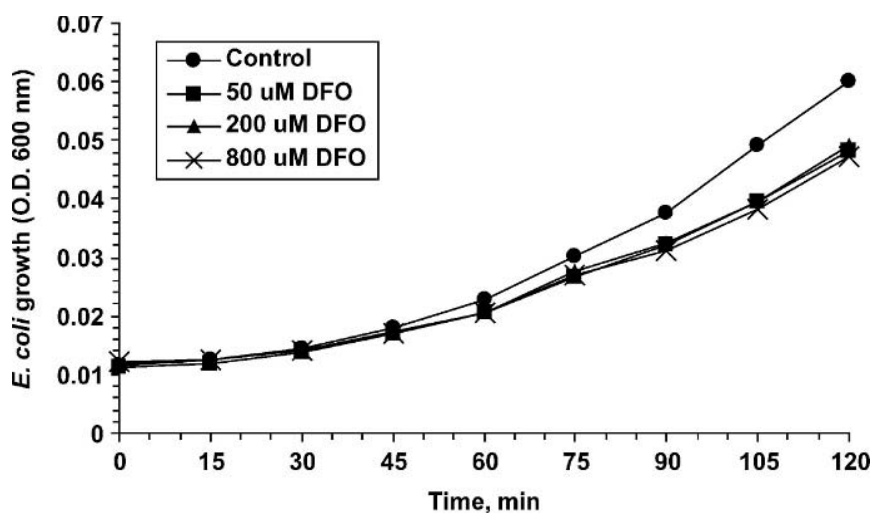

Figure 3. Effect of different concentrations of deferoxamine (DFO) on Escherichia coli growth. Bacterial growth was determined by measurements of optical density at $600 \mathrm{~nm}$. The values represent the means of 3 independent experiments in which each DFO concentration was tested in triplicate. Bacterial growth in the presence of DFO was significantly slower $(P<0.05)$ from time point $60 \mathrm{~min}$ for all concentrations tested compared with the control.

\section{DISCUSSION}

In the first part of the study, an in vitro model of coculture of MAC-T cells and activated bovine PMN was used to investigate whether some antioxidants and ion chelators were able to prevent PMN-induced mammary epithelial damages. Our results showed that BDA and DMTU did not protect MAC-T cells against oxidative damages at the doses used. On the other hand, addition of CAT, DFO, and GEE led to a dose-dependent reduction of PMN-induced cell cytotoxicity, as demonstrated by the significant decrease in released $\mathrm{LDH}$ and by visual observation of the integrity of MAC-T cells under the microscope.

The exact mechanism by which bovine epithelial cells are damaged in mastitis is still not fully understood. It is well known that PMN, when activated, release

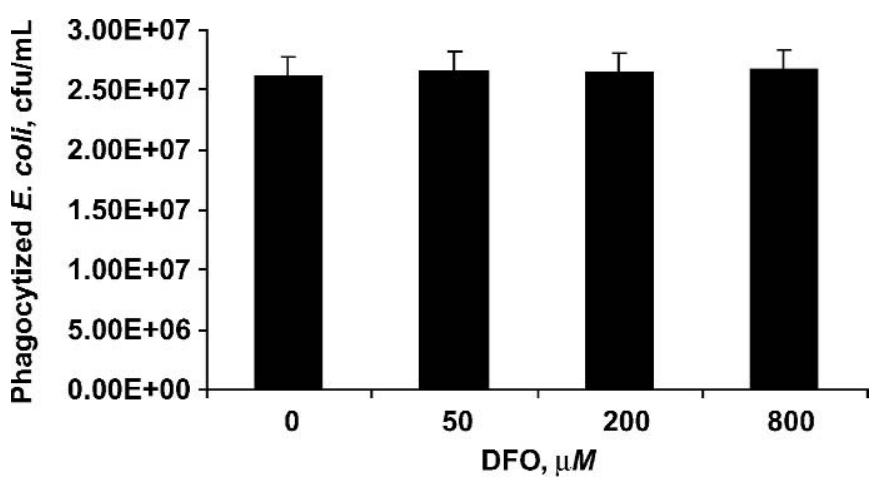

Figure 4. Effect of deferoxamine (DFO) on neutrophils' ability to phagocytose Escherichia coli. The results represent the number of ingested bacteria $(\mathrm{cfu} / \mathrm{mL})$ and are expressed as means \pm SEM. bactericidal molecules such as ROS that eliminate microbes but are also harmful toward host cells. Using inflammation models, PMN have been shown to induce tissue damage through the release of ROS, proteases, and lysozymes (Varani and Ward, 1994; Ginsburg, 1998; Poch et al., 1999). In the mammary gland, prolonged diapedesis of PMN induces damage to mammary parenchyma tissue, which results in decreased milk production (Sordillo et al., 1997). In the present study, activated PMN induced considerable cytotoxicity in MAC-T cells, whereas incubation of these cells with nonactivated PMN did not cause any cytotoxicity. In our assay, activation of the oxidative metabolism of PMN was achieved by supplementing culture medium with PMA $(1 \mu \mathrm{g} / \mu \mathrm{L})$, whereas LPS $(1 \mu \mathrm{g} / \mu \mathrm{L})$ was added to prevent PMN from undergoing spontaneous apoptosis and to extend their life span (Lee et al., 1993). Unlike PMN, MAC-T cells were not affected by the presence of LPS and PMA as demonstrated by the absence of significant LDH release. These results are in agreement with previous observations (Ledbetter et al., 2001; Boulanger et al., 2002).

At the concentrations tested, DMTU was unable to reduce cell death. Adding BDA to the coculture only induced a slight level of protection at a very high concentration. However, this concentration would not be achievable in vivo because incubation of $1000 \mu M$ of BDA with MAC-T cells alone was toxic to MAC-T cells as shown by an increase in cytotoxicity levels. In general, DMTU is regarded as a free-radical scavenger. However, it has also been reported that DMTU could act as a copper chelator (Hanna and Mason, 1992) like BDA. Therefore, the relative ineffectiveness of both DMTU and BDA in preventing cell cytotoxicity suggests that free copper is not an important mediator in PMNinduced bovine epithelial cell damage. These data might suggest that the biodistribution of DMTU in our cell system was not appropriate to react with the hydroxyl radicals.

In the past decades, it has been demonstrated both in vitro and in vivo that tea and tea polyphenols exhibit strong antioxidant properties, including scavenging of oxygen radicals (hydrogen peroxide, hydroxyl radicals) and lipid radicals (Salah et al., 1995). Catechin is a flavonoid polyphenol found in tea that has been reported to be 20 times more potent than vitamin $\mathrm{C}$ as an antioxidant (Craig, 1999). Our results show that CAT reduced LDH release and thus cell cytotoxicity by $77 \%$ at $25 \mu M$ and by $100 \%$ at $50 \mu M$ when added to the coculture of activated PMN and MAC-T cells. This protective effect was confirmed by visual observation of acridine orange-stained MAC-T cells. This is consistent with the findings of an in vitro study conducted by Chen et al. (2002), who observed that tea catechin treatment 
significantly increased cell viability and decreased lipid peroxidation levels in lead-exposed cells in which the damage was mediated by ROS. Furthermore, green tea polyphenols were found to block LPS-induced lethality when given orally to BALB/c mice before an intraperitoneal injection of LPS (Yang et al., 1998). Taken together, these results suggest that catechin may be useful as a therapy in various inflammatory processes such as mastitis.

Under physiological conditions, a small amount of ROS is produced due to the metabolic processes of all aerobic cells. These ROS are neutralized by endogenous antioxidants such as GSH. Several studies have linked GSH to the ability of a cell to resist the deleterious effect of ROS (Singhal and Jain, 2000), and more specifically $\mathrm{H}_{2} \mathrm{O}_{2}$ (Seo et al., 2004). Indeed, reduced GSH is the substrate of the $\mathrm{H}_{2} \mathrm{O}_{2}$-removing enzyme glutathione peroxidase. In our model, GEE was used to increase the endogenous pool of GSH in MAC-T cells because GEE can easily enter cells (unlike GSH). Once inside the cells, GEE is rapidly hydrolyzed to yield reduced glutathione (Anderson et al., 1985). Our results show that GEE was able to prevent PMN-induced damage in MAC-T cells, with an $83 \%$ decrease in cell cytotoxicity when cocultures were supplemented with 50 or 100 $\mu M$ GEE. This protective effect was also confirmed by observation of cells stained with acridine orange. Furthermore, no abnormality in cellular morphology was observed. The protective effect of GEE has been reported by others; for example, Morris et al. (1995) demonstrated that GEE was able to attenuate LPS-induced injury of cultured bovine pulmonary artery endothelial cells. Therefore, in our coculture model, it is likely that GEE was able to increase the endogenous pool of GSH in MAC-T cells and, thus, enhanced the ability of these cells to detoxify $\mathrm{H}_{2} \mathrm{O}_{2}$. This explanation is supported by a study performed by Michiels et al. (1994) in which the rate of $\mathrm{H}_{2} \mathrm{O}_{2}$ elimination was shown to increase linearly with GSH concentration when GSH was unable to saturate glutathione peroxidase. Knowing that $\mathrm{H}_{2} \mathrm{O}_{2}$ is the central component from which the majority of ROS are produced, it is not surprising to observe a decrease in cell cytotoxicity caused by PMN oxidative burst. These data suggest that $\mathrm{H}_{2} \mathrm{O}_{2}$ is an important mediator of tissue damage in bovine mammary epithelial cells and that an efficient scavenging of $\mathrm{H}_{2} \mathrm{O}_{2}$ could significantly reduce cellular damage when oxidative stress arises from massive PMN activation.

The present study also shows that the iron chelator DFO reduced the release of LDH by MAC-T cells in a concentration-dependent manner. Acridine orange staining revealed an intact monolayer of MAC-T cells in contrast with cells incubated with activated PMN alone. Furthermore, when MAC-T cells were incubated with DFO alone, no significant increase in LDH release was observed after $24 \mathrm{~h}$, showing that DFO was not intrinsically toxic. This is consistent with another in vitro study performed on human endothelial cells, which reported that DFO did not increase cell susceptibility to exogenous oxidants after $48 \mathrm{~h}$ (Fratti et al., 1998). Furthermore, DFO has been shown to be protective in various models of ROS-induced cellular injury as reviewed by van Asbeck (1990). Additionally, DFO was found to react with oxygen products released by PMN following PMA activation (Soriani et al., 1993). In our model, the protective effect of DFO is probably accomplished by chelation of free iron, which then becomes unavailable for the Fenton reactions and therefore hydroxyl radical generation. This standpoint is reinforced by a study indicating that DFO completely prevented iron from catalyzing Haber-Weiss and Fenton reactions (Gutteridge et al., 1979). Because hydroxyl radical is thought to be the most harmful radical for tissue damage, it is not surprising to see a lower level of cytotoxicity in DFO-treated cells. Additionally, it has been reported that pretreating endothelial cells with DFO is sufficient to decrease PMN-induced damage; this also suggests that target cells and not PMN provide the necessary iron (Gannon et al., 1987). Therefore, DFO may be a useful chemical for preventing PMN-induced cell damage.

The second part of the study evaluated the in vitro effect of DFO on bacterial growth $(E$. coli $)$ and the ability of PMN to ingest $E$. coli. Our results showed significant inhibition of the bacterial growth rate when DFO was added to the culture medium. The difference between the control and the DFO-treated $E$. coli became significant after $60 \mathrm{~min}$ of incubation for all concentrations tested ( 50 to $800 \mu M$ ). Iron is known to be essential to all living organisms, including $E$. coli. The DFO-induced inhibition of bacterial growth observed in our experiment can be compared with the findings in an in vivo study with cows, in which addition of DFO to milk from LPS-infused quarters depressed $E$. coli growth (Lohuis et al., 1988). The same study also demonstrated that $E$. coli growth was promoted by adding iron to milk. Although some pathogens such as Staphylococcus aureus can use DFO as an iron source (Diarra et al., 2002), it appears that iron chelated by DFO is unavailable for $E$. coli. However, as this bacterium possesses at least 7 iron-acquisition systems and they can increase their capacity to assimilate iron when an iron deficiency occurs, it is possible that the observed inhibition may be transient and last only as long as it takes $E$. coli to modify its metabolism to activate other mechanisms of iron uptake.

In our assay, phagocytosis was demonstrated by the decreased number of bacteria remaining in the super- 
natant of control wells compared with the number of bacteria initially added. Our results did not show a significant effect of DFO on PMN ability to ingest $E$. coli because the ability of PMN to ingest bacteria was the same with or without DFO. This suggests that iron chelation by DFO did not induce an iron deficiency in PMN because phagocytosis requires energy, and production of energy through oxygen metabolism requires iron as a transition metal (Macdonald et al., 1985). There are contradictory reports in the literature regarding the effect of DFO on phagocytosis. Indeed, some studies have reported that incubating PMN (from healthy humans) with DFO induced progressive alteration of phagocytosis performance (Cantinieaux et al., 1990), whereas others have concluded that DFO enhanced phagocytic function (van Asbeck et al., 1984; Ewald et al., 1994). These differences are probably caused by different experimental procedures that include or exclude serum, and by the use of different bacteria (Staphylococcus aureus, Yersinia enterolitica, and $E$. coli).

The effect of DFO on the internal bactericidal activity of bovine PMN was not specifically investigated in the present study but plating of PMN lysate showed no significant bacterial growth, suggesting that killing ability of PMN was not affected. Using human PMN, some studies have demonstrated in vitro that DFO might reduce the microbicidal activity of phagocytes by completely preventing iron from catalyzing the HaberWeiss reaction. Consequently, the amount of ROS produced by PMN may not be sufficient to complete the intracellular killing (Ewald et al., 1994). Whether DFO interferes with the ability of bovine PMN to kill ingested bacteria still needs to be evaluated.

In conclusion, activated bovine PMN caused severe damage to MAC-T cells. These deleterious effects could be inhibited partially or totally through supplementation with antioxidants (catechin, deferoxamine, or glutathione ethyl ester), a finding that suggests that ROS are a major cause of PMN-mediated tissue damage during mastitis. In addition, the protective effect of these antioxidants was observed at concentrations that would be reachable by intramammary infusion. Therefore, the use of antioxidants in treating bovine mastitis is worth investigating as they may aid in preventing mammary tissue damage. Additionally, some antioxidants such as DFO may mitigate the impact of $E$. coli invasion by inhibiting bacterial growth.

\section{ACKNOWLEDGMENTS}

This work was supported by NOVALAIT Inc., Fonds Québécois de la Recherche sur la Nature et les Technologies, and the Ministère de l'Agriculture, des Pêcheries et de l'Alimentation du Québec. We also thank McGill University and Agriculture and Agri-Food Canada.

\section{REFERENCES}

Anderson, M. E., F. Powrie, R. N. Puri, and A. Meister. 1985. Glutathione monoethyl ester: Preparation, uptake by tissues, and conversion to glutathione. Arch. Biochem. Biophys. 239:538-548.

Boulanger, V., X. Zhao, and P. Lacasse. 2002. Protective effects of melatonin and catalase in bovine neutrophil-induced model of mammary cell damage. J. Dairy Sci. 85:562-569.

Cantinieaux, B., C. Hariga, A. Fertster, M. Toppet, and P. Fondu. 1990. Desferrioxamine improves neutrophil phagocytosis in thalassemia major. Am. J. Hematol. 35:13-17.

Carlson, G. P., and J. J. Kaneko. 1973. Isolation of leukocytes from bovine peripheral blood. P.S.E.B.M. 142:853-856.

Chen, L., X. Yang, H. Jiao, and B. Zhao. 2002. Tea catechins protect against lead-induced cytotoxicity, lipid peroxidation, and membrane fluidity in HepG2 cells. Toxicol. Sci. 69:149-156.

Craig, W. J. 1999. Health-promoting properties of common herbs. Am. J. Clin. Nutr. 70(Suppl. 3):491S-499S.

Dehne, N., J. Lautermann, F. Petrat, U. Rauen, and H. de Groot. 2001. Cisplatin ototoxicity. Involvement of iron and enhanced formation of superoxide anion radicals. Toxicol. Appl. Pharmacol. $174: 27-34$.

Diarra, M. S., D. Petitclerc, and P. Lacasse. 2002. Response of Staphylococcus aureus to exogenous iron. J. Dairy Sci. 85:2141-2148.

Emerit, J., C. Beaumont, and F. Trivin. 2001. Iron metabolism, free radicals, and oxidative injury. Biomed. Pharmacother. 55:333339.

Ewald, J. H., J. Heesemann, H. Rüdiger, and I. B. Autenrieth. 1994. Interaction of polymorphonuclear leukocytes with Yersinia enterolitica: Role of the Yersinia virulence plasmid and modulation by the iron-chelator desferrioxamine Br. J. Infect. Dis. 170:140-150.

Fischer, U., M. Ototake, and T. Nakanishi. 1998. In vitro cell-mediated cytotoxicity against allogeneic erythrocytes in ginbuna crucian carp and goldfish using a non-radioactive assay. Dev. Comp. Immunol. 22:195-206.

Fratti, R. A., P. H. Belanger, M. A. Ghannoum, J. E. Edwards Jr., and S. G. Filler. 1998. Endothelial cell injury caused by Candida albicans is dependent on iron. Infect. Immun. 66:191-196.

Gannon, D. E., J. Varani, S. H. Phan, J. H. Ward, J. Kaplan, G. O. Till, R. H. Simon, U. S. Ryan, and P. A. Ward. 1987. Source of iron in neutrophil-mediated killing of endothelial cells. Lab. Invest. 57:37-44.

Ginsburg, I. 1998. Could synergistic interactions among reactive oxygen species, proteinases, membrane-perforating enzymes, hydrolases, microbial hemolysins and cytokines be the main cause of tissue damage in infectious and inflammatory conditions? Med. Hypotheses 51:337-346.

Gutteridge, J. M., R. Richmond, and B. Halliwell. 1979. Inhibition of the iron-catalysed formation of hydroxyl radicals from superoxide and of lipid peroxidation by desferrioxamine. Biochem. J. 184:469-472.

Hanna, P. M., and R. P. Mason. 1992. Direct evidence for inhibition of free radical formation from $\mathrm{Cu}(\mathrm{I})$ and hydrogen peroxide by glutathione and other potential ligands using the EPR spin-trapping technique. Arch. Biochem. Biophys. 295:205-213.

Huynh, H. T., G. Robitaille, and J. D. Turner. 1991. Establishment of bovine mammary epithelial cells (MAC-T): An in vitro model for bovine lactation. Exp. Cell Res. 197:191-199.

Karlsson, A., J. B. Nixon, and L. C. McPhail. 2000. Phorbol myristate acetate induces neutrophil NADPH-oxidase activity by two separate signal transduction pathways: Dependent or independent of phosphatidylinositol 3-kinase. J. Leukoc. Biol. 67:396-404.

Kennedy, K. A., and N. L. Lane. 1994. Effect of in vivo hyperoxia on the glutathione system in neonatal rat lung. Exp. Lung Res. 20:73-83.

Ledbetter, T. K., M. J. Paape, and L. W. Douglas. 2001. Cytotoxic effects of peroxynitrite, polymorphonuclear neutrophils, free radi- 
cal scavengers, inhibitors of myeloperoxidase, and inhibitors of nitric oxide synthase on bovine mammary secretory epithelial cells. Am. J. Vet. Res. 62:286-293.

Lee, A., M. K. B. Whyte, and C. H. Haslett. 1993. Inhibition of apoptosis and prolongation of neutrophil functional longevity by inflammatory mediators. J. Leukoc. Biol. 54:283-288.

Lohuis, J. A., W. Van Leeuwen, J. H. Verheijden, J. A. Smit, A. Brand, and A. S. Van Miert. 1988. Growth of Escherichia coli in whole and skim milk from endotoxin-induced mastitic quarters: In vitro effects of deferoxamine, zinc, and iron supplementation. J. Dairy Sci. 71:2772-2781.

Macdonald, V. W., S. Charache, and P. J. Hathaway. 1985. Iron deficiency anemia: Mitochondrial alpha-glycerophosphate dehydrogenase in guinea pig skeletal muscle. J. Lab. Clin. Med. 105:11-18.

Michiels, C., M. Raes, O. Toussaint, and J. Remacle. 1994. Importance of Se-glutathione peroxidase, catalase, and $\mathrm{Cu} / \mathrm{Zn}$-SOD for cell survival against oxidative stress. Free Radic. Biol. Med. 17:235-248.

Miller, E. J., and R. K. Rhodes. 1982. Preparation and characterization of the different types of collagen. Methods Enzymol. 82:33-64.

Morris, P. E., A. P. Wheeler, and B. O. Meyrick. 1995. Escherichia coli endotoxin-mediated endothelial injury is modulated by glutathione ethyl ester. J. Infect. Dis. 172:1119-1122.

Oikawa, S., and S. Kawanishi. 1996. Site-specific DNA damage induced by NADH in the presence of copper(II): Role of active oxygen species. Biochemistry 35:4584-4590.

Poch, B., F. Gansauge, B. Rau, U. Wittel, S. Gansauge, A. K. Nüssler, M. Schoenberg, and H. G. Beger. 1999. The role of polymorphonuclear leukocytes and oxygen-derived free radicals in experimental acute pancreatitis: Mediators of local destruction and activators of inflammation. FEBS Lett. 461:268-272.

Salah, N., N. J. Miller, G. Paganga, L. Tijburg, G. P. Bolwell, and C. Rice-Evans. 1995. Polyphenolic flavanols as scavengers of aqueous phase radicals and as chain-breaking antioxidants. Arch. Biochem. Biophys. 322:339-346.

Seo, Y. J., J. W. Lee, E. H. H. K. Lee, H. W. Lee, and Y. H. Kim. 2004. Role of glutathione in the adaptive tolerance to $\mathrm{H}_{2} \mathrm{O}_{2}$. Free Radic. Biol. Med. 37:1272-1281.
Singhal, R. K., and A. Jain. 2000. Glutathione ethyl ester supplementation prevents mortality in newborn rats exposed to hyperoxia. Biol. Neonate 77:261-266.

Smith, J. A. 1994. Neutrophils, host defense, and inflammation: A double-edged sword. J. Leukoc. Biol. 56:672-686.

Sordillo, L. M., K. Shafer-Weaver, and D. DeRosa. 1997. Immunobiology of the mammary gland. J. Dairy Sci. 80:1851-1865.

Soriani, M., S. Mazzuca, V. Quaresima, and M. Minetti. 1993. Oxidation of desferrioxamine to nitroxide free radical by activated human neutrophils. Free Radic. Biol. Med. 14:589-599.

Sprong, R. C., J. M. Aarsman, J. F. L. M. van Oirschot, and B. S. van Asbeck. 1997. Dimethylthiourea protects rats against gramnegative sepsis and decreases tumor necrosis factor and nuclear factor $\mathrm{kB}$ activity. J. Lab. Clin. Med. 129:470-481.

van Asbeck, B. S. 1990. Oxygen toxicity: Role of hydrogen peroxide and iron. Adv. Exp. Med. Biol. 264:235-246.

van Asbeck, B. S., J. J. Marx, A. Struyvenberg, J. H. van Kats, and J. Verhoef. 1984. Deferoxamine enhances phagocytic function of human polymorphonuclear leukocytes. Blood 63:714-720.

Varani, J., and P. A. Ward. 1994. Mechanisms of neutrophil-dependent and neutrophil-independent endothelial cell injury. Biol. Signals 3:1-14.

Verhaegh, G. W., M.-J. Richard, and P. Hainaut. 1997. Regulation of p53 by metal ions and by antioxidants: Dithiocarbamate downregulates p53 DNA-binding activity by increasing the intracellular level of copper. Mol. Cell. Biol. 17:5699-5706.

Visseren, F. L. J., M. S. A. Verkerk, T. van der Bruggen, J. J. M. Marx, B. S. van Asbeck, and R. J. A. Diepersloot. 2002. Iron chelation and hydroxyl radical scavenging reduce the inflammatory response of endothelial cells after injection with Chlamydia pneumoniae or influenza. Eur. J. Clin. Invest. 32(Suppl.1):84-90.

Weiss, S. J. 1989. Tissue destruction by neutrophils. N. Engl. J. Med. 320:365-376.

Yang, F., W. J. de Villiers, C. J. McClain, and G. W. Varilek. 1998. Green tea polyphenols block endotoxin-induced tumor necrosis factor-production and lethality in a murine model. J. Nutr. 128:2334-2340. 